\title{
EXTENSIONS OF THE HEISENBERG-WEYL INEQUALITY
}

\author{
H. P. HEINIG and M. SMITH \\ Department of Mathematical Sciences \\ McMaster University \\ Hamilton, Ontario \\ L8S 4K1, Canada \\ (Received February 20, 1985)
}

\begin{abstract}
In this paper a number of generalizations of the classical Heisenberg-Weyl uncertainty inequality are given. We prove the n-dimensional Hirschman entropy inequality (Theorem 2.1) from the optimal form of the Hausdorff-Young theorem and deduce a higher dimensional uncertainty inequality (Theorem 2.2). From a general weighted form of the Hausdorff-Young theorem, a one-dimensional weighted entropy inequality is proved and some weighted forms of the Heisenberg-Weyl inequalities are given.

KEY WORDS AND PHRASES. Uncertainty Inequality, Fourier Transform, Variance, Entropy Hausdorff-Young Inequality, Weighted Norm Inequalities.

1980 AMS SUBJECT CLASSIFICATION CODE. $26 D 10,42 A 38$.
\end{abstract}

1. INTRODUCTION.

Let $\hat{\mathrm{f}}$ be the Fourier transform of $\mathrm{f}$ defined by $\hat{f}(x)=\int e^{-2 \pi i x y} f(y) d y, \quad x \in \mathbb{R}$.

If $f \in L^{2}(\mathbb{R})$ with $L^{2}$-norm $\|f\|_{2}=1$, then by Plancherel's theorem $\|\hat{\mathrm{f}}\|_{2}=1$, so that $|\mathrm{f}(\mathrm{x})|^{2}$ and $|\hat{\mathrm{f}}(\mathrm{y})|^{2}$ are probability trequency functıons. The variance of a $\mathrm{p}$ covability frequency function $\mathrm{g}$ is defined by

$$
\mathrm{V}[\mathrm{g}]=\int_{\mathbb{R}}(\mathrm{x}-\mathrm{m})^{2} \mathrm{~g}(\mathrm{x}) \mathrm{dx} \quad \text { where } \quad \mathrm{m}=\int_{\mathbb{R}} \mathrm{xg}(\mathrm{x}) \mathrm{dx}
$$

is the mean. With these notations, the Heisenberg uncertainty principle of quantum mechanics can be stated in terms of the Fourier transform by the inequality $V\left[|f|^{2}\right] V\left[|\hat{f}|^{2}\right]>\left(16 \pi^{2}\right)^{-1}$.

In the sequel, we assume without loss of generality that the mean $m=0$. If $g$ is a probability frequency function, then the entropy of $g$ is defined by

$$
E[g]=\int_{\mathbb{R}} g(x) \log g(x) d x .
$$

With $\mathrm{f}$ as above, Hirschman [1] proved that

$$
E\left[|f|^{2}\right]+E\left[|\hat{f}|^{2}\right] \leqslant E_{H}
$$

with $E_{H}=0$, and suggested that (1.2) holds with $E_{H}=10 g$ 2-1. If $E_{H}$ has that form, then by an inequality of Shannon and Weaver [2] it follows that (1.2) implies (1.1). Using the Babenko-Beckner optimal form of the Hausdorff-Young inequality ([3])

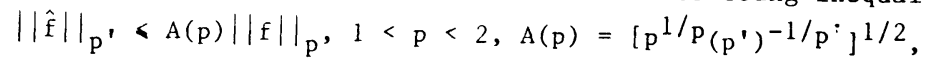


in Hirschman's proof of (1.2), then as Beckner [3] noted, (1.2) holds with $E_{H}=10 g$ 2-1. A modest extension of $(1.1)$ is obtained as follows: Let $f$ on $\mathbb{R}$ be differentiable, such that $f(0)=0$. Then Hölder's and Hardy's inequality [4, Theorem 3.27] yield with $1<p \leqslant 2$

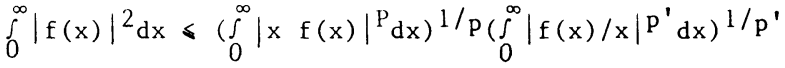

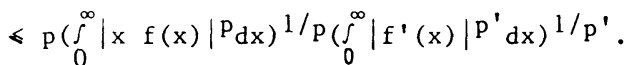

Applying this estimate also to $f(-x)$, then

$$
\begin{aligned}
& \|\left. f\right|_{2} ^{2}=\int_{0}^{\infty}|f(x)|^{2} d x+\int_{0}^{\infty}|f(-x)|^{2} d x
\end{aligned}
$$

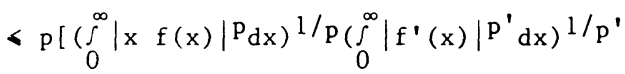

$$
\begin{aligned}
& \left.+\left(\int_{0}^{\infty}|\mathrm{x} f(-\mathrm{x})|^{\mathrm{P}} \mathrm{dx}\right)^{1 / \mathrm{P}}\left(\int_{0}^{\infty}\left|\mathrm{f}^{\prime}(-\mathrm{x})\right|^{\mathrm{P}^{\prime} \mathrm{dx}}\right)^{1 / \mathrm{P}^{\prime}}\right] \\
& \leqslant \mathrm{p}\left(\int_{-\infty}^{\infty}|\mathrm{x} f(\mathrm{x})|^{\mathrm{P}} \mathrm{dx}\right)^{1 / \mathrm{P}}\left(\int_{-\infty}^{\infty}\left|\mathrm{f}^{\prime}(\mathrm{x})\right|^{\mathrm{P}^{\prime} \mathrm{dx}}\right)^{1 / \mathrm{p}^{\prime},}
\end{aligned}
$$

where the last inequality follows from Hölder's inequality. Now by (1.3) and the fact that $\hat{\mathrm{f}}^{\prime}(\mathrm{y})=2 \pi \mathrm{iy} \hat{\mathrm{f}}(\mathrm{y})$ we obtain

THEOREM 1.l. If $\mathrm{f} \in \mathrm{S}(\mathbb{R})$ and $\mathrm{f}(0)=0$, then for $\mathrm{l}<\mathrm{p} \leqslant 2$

$$
\|\mathrm{f}\|_{2}^{2} \leqslant 2 \pi \text { p } A(\mathrm{p})\|\mathrm{xf}\|_{\mathrm{p}}\|\mathrm{y} \hat{f}\|_{\mathrm{p}} \text {. }
$$

Note that the constant in $(1.4)$ is slightly better than that in $[4,51.4]$ but unlikely best possible.

The purpose of this paper is to give extensions of the Heisenberg-Weyl inequality (1.1). In the next section a new proof of the entropy inequality (1.2) for functions on $\mathbb{R}^{\mathrm{n}}$ is given and an $\mathrm{n}$-dimensional Heisenberg-Weyl inequality is deduced. The $\mathrm{n}$ dimensional generalization of inequality (1.4) is also given in the next section. The two inequalities are quite different, even in the case $p=2$, but depend strongly on the sharp Hausdorff-Young inequality. In the third section a weighted form of the Heisenberg-Weyl inequality in one dimension is obtained from a weighted form of the Hausdorff-Young inequality $([5][6][7][8])$. Unlike the constant $A(p)$ in (1.3) the constant of the weighted Hausdorff-Young inequality (3.3) of (Theorem 3.1) is far from sharp. If the constant is not too large, then a weighted form of Hirschman's entropy inequality can also be given, from which another uncertainty inequality is deduced.

Throughout, $\mathrm{p}^{\prime}=\mathrm{p} /(\mathrm{p}-1)$, with $\mathrm{p}^{\prime}=\infty$ if $\mathrm{p}=1$, is the conjugate index of $\mathrm{p}$, and similarly for other letters. $S\left(\mathbb{R}^{n}\right)$ is the Schwartz class of slowly increasing functions on $\mathbb{R}^{\mathrm{n}}$. We say $\mathrm{g}$ is in the weighted $\mathrm{L}_{\mathrm{w}}^{\mathrm{r}}$-space with weight $w$, if $w g \varepsilon L^{r}$ and norm $\|\mathrm{g}\|_{\mathrm{r}, \mathrm{w}}=\|\mathrm{wg}\|_{\mathrm{r}}$. If $\mathrm{x} \varepsilon \mathbb{R}^{\mathrm{n}}$, then $\mathrm{x}=\left(\mathrm{x}_{1}, \mathrm{x}_{2}, \ldots, \mathrm{x}_{\mathrm{n}}\right)$ and $\mathrm{dx}=\mathrm{dx}_{1} \ldots \mathrm{dx}_{\mathrm{n}}$ the $\mathrm{n}-$ dimensional Lebesgue measure. $f_{i}(x), x \in \mathbb{R}^{n}$ denotes the partial derivative of $f$ with respect to the $i^{\text {th }}$ component and $f_{i j}=\left(f_{i}\right)_{j}$. The letter $C$ denotes a constant which may be different at different occurrences, but is independent of $\mathrm{f}$.

\section{THE HIRSCHMAN INEQUALITY.}

The Fourier transform of $f$ on $\mathbb{R}^{n}$ is given by

$$
\hat{f}(x)=\int_{\mathbb{R}^{n}} e^{-2 \pi i x \cdot y} f(y) d y, \quad x \in \mathbb{R}^{n}, \quad x \cdot y=x_{1} y_{1}+\ldots+x_{n} y_{n i} ;
$$

and the entropy of a function on $\mathbb{R}^{n}$ is defined as before with $\mathbb{R}$ replaced by $\mathbb{R}^{\mathrm{n}}$. We shall need the following well known result (c.f. [9; $\$ 13.32$ ii]): 
If $\int_{x} d \mu=1$, then

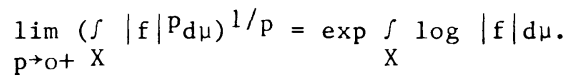

Using this fact we obtain easily the n-dimensional form of Hirschman's inequality (1.2)

THEOREM 2.l. If $\mathrm{f} \in \mathrm{L}^{2}\left(\mathbb{R}^{\mathrm{n}}\right)$ such that $\|\mathrm{f}\|_{2}=\| \hat{\mathrm{f}}||_{2}=1$, then

$$
E\left[|f|^{2}\right]+E\left[|\hat{f}|^{2}\right] \leqslant n[\log 2-1],
$$

whenever the left side has meaning.

PROOF. Let $f \in\left(L^{1} \Gamma_{i} L^{2}\right)\left(\mathbb{R}^{n}\right)$, then $f \in L^{P}\left(\mathbb{R}^{n}\right), 1<p<2$, and by the $n-d i m e n-$ sional form of the sharp Hausdorff-Young inequality [3] (that is, (1.3) with A(p) replaced by $[A(p)]^{n}$ ) we obtain with $p=2-r, r>0$ and $p^{\prime}=2-r^{\prime}, r^{\prime}<0$

$$
\left(\int_{\mathbb{R}^{n}}|\hat{f}(y)|^{2-r^{\prime}} d y\right)^{-1 / r^{\prime}} \leqslant\left[\frac{(2-r)^{1 / 2 r)}}{\left(2-r^{\prime}\right)^{-1 /\left(2 r^{\prime}\right)}}\right]^{n}\left(\int_{\mathbb{R}^{n}}|f(x)|^{2-r} d x\right)^{1 / r} .
$$

Now let $\mathrm{d} \hat{\mu}=|\hat{\mathrm{f}}(\mathrm{y})|^{2} \mathrm{dy}$ and $\mathrm{d} \mu=|\mathrm{f}(\mathrm{x})|^{2} \mathrm{dx}$, then $\int_{\mathbb{R}^{\mathrm{n}}} \mathrm{d} \hat{\mu}=\int_{\mathbb{R}^{n}} \mathrm{~d} \mu=1$, so that the inequality becomes

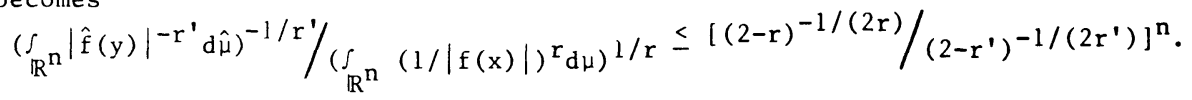

But as $r \rightarrow$ ot, $-r^{\prime} \rightarrow$ o+, so that by $(2.1)$

$$
\begin{aligned}
& \exp \left(\int_{\mathbb{R}^{n}} \log |\hat{\mathrm{f}}(\mathrm{y})| \mathrm{d} \hat{\mu}\right) / \\
& \exp \left(\int_{\mathbb{R}^{n}} \log \left(|\mathrm{f}(\mathrm{x})|^{-1}\right) \mathrm{d} \mu\right) \\
& =\exp \left(\int_{\mathbb{R}^{n}}|\hat{f}(y)|^{2} \log |\hat{f}(y)| d y+\int_{\mathbb{R}^{n}}|f(x)|^{2} \log |f(x)| d x\right) \leqslant \frac{1 i m}{r \rightarrow 0} \frac{(2-r)^{\frac{n}{(2 r)}}}{\left(2-r^{\prime}\right)^{-n /\left(2 r^{\prime}\right)}} \\
& =2^{n / 2} e^{-n / 2} .
\end{aligned}
$$

Taking logarithms on both sides we get

$$
\int_{\mathbb{R}^{n}}|\hat{f}(y)|^{2} \log |\hat{f}(y)| d y+\int_{\mathbb{R}^{n}}|f(x)|^{2} \log |f(x)| d x<\frac{n}{2}[\log 2-1]
$$

and this implies (2.2) in the case $f \varepsilon\left(L^{l} \cap L^{2}\right)\left(\mathbb{R}^{n}\right)$.

If $f \varepsilon L^{2}$ the result is obtained as in [1] only now one takes for $\omega_{T}, \omega_{\varepsilon}(x)=$ $\mathrm{e}^{-\pi \varepsilon|\mathrm{x}|^{2}}$ and for $\Omega_{\mathrm{T}}, \hat{\omega}_{\varepsilon}(\mathrm{y})=\varepsilon^{-\mathrm{n} / 2} \mathrm{e}^{-\pi|\mathrm{y}|^{2}} / \varepsilon$. We omit the details.

If $|g| \varepsilon L^{2}(\mathbb{R})$ is a probability frequency function, then the relation between entropy and variance is expressed by $E\left[|g|^{2}\right] \geqslant-\frac{1}{2}-\frac{1}{2} \log \left(2 \pi V\left[|g|^{2}\right]\right)([2 ; p$. 55-56]). The n-dimensional form of this inequality is given in the following lemma:

LEMMA 2.1. ([2; p. 56-57]). Let $g \in L^{2}\left(\mathbb{R}^{n}\right)$ with $\|g\|_{2}=1$. If $B=\left(b_{i j}\right)$ is the matrix with entries

then

$$
b_{i j}=v\left[|g|^{2}\right]=\int_{\mathbb{R}^{n}} x_{i} x_{j}|g(x)|^{2} d x, \quad i, j=1,2, \ldots, n \text {; }
$$

where $\left|b_{i j}\right|=\operatorname{det} B$.

$$
E\left[|g|^{2}\right] \geqslant \frac{n}{2} \log \left(2 \pi\left|b_{i j}\right|^{1 / n}\right)-n / 2
$$

Using the lemma and Theorem 2.1, we easily establish an n-dimensional extension of the Heisenberg-Weyl inequality.

THEOREM 2.2. Let $\mathrm{f} \in \mathrm{L}^{2}\left(\mathbb{R}^{\mathrm{n}}\right)$ with $\|\mathrm{f}\|_{2}=|| \hat{\mathrm{f}} \|_{2}=1$ and

$$
b_{i j}=\int_{\mathbb{R}^{n}} x_{i} x_{j}|f(x)|^{2} d x, \quad \hat{b}_{i j}=\int_{\mathbb{R}} y_{i} y_{j}|\hat{f}(y)|^{2} d y,
$$


$i, j=1,2, \ldots n$; be the entries of the matrices $B$ and $\hat{B}$ respectively, then $(\operatorname{det} B)(\operatorname{det} \hat{B}) \geqslant\left(16 \pi^{2}\right)^{-n}$.

PROOF. By (2.2) and Lemma 2.1,

$$
\begin{aligned}
& n[\log 2-1] \geqslant E\left[|f|^{2}\right]+E\left[|\hat{f}|^{2}\right] \\
& \geqslant-\frac{n}{2} \log \left(2 \pi\left|b_{i j}\right|^{1 / n}\right)-\frac{n}{2} \log \left(2 \pi\left|\hat{b}_{i j}\right|^{1 / n}\right)-n,
\end{aligned}
$$

so that

$$
\log 2 \geqslant-\frac{1}{2} \log \left(4 \pi^{2}\left|b_{i j}\right|^{1 / n}\left|\hat{b}_{i j}\right|^{1 / n}\right)
$$

But then

$$
4 \geqslant 1 /\left[(\operatorname{det} B)^{1 / n}(\operatorname{det} \hat{B})^{1 / n_{4}} \pi^{2}\right],
$$

which implies the result.

Clearly, if $\mathrm{n}=1$ we obtain at once $(1 \cdot 1)$. If $\mathrm{n}=2$ then

$$
B=\left(\begin{array}{ll}
b_{11} & b_{12} \\
b_{21} & b_{22}
\end{array}\right)=\left\{\begin{array}{ll}
f_{\mathbb{R}^{2}} \mathrm{x}_{1}^{2}|\mathrm{f}|^{2} \mathrm{dx}, & \int_{\mathbb{R}^{2}} \mathrm{x}_{1} \mathrm{x}_{2}|\mathrm{f}|^{2} \mathrm{dx} \\
\int_{\mathbb{R}} \mathrm{x}_{2} \mathrm{x}_{1}|\mathrm{f}|^{2} \mathrm{dx}, & \int_{\mathbb{R}^{2}} \mathrm{x}_{2}^{2}|\mathrm{f}|^{2} \mathrm{dx}
\end{array}\right\},
$$

with a similar expression for $\hat{B}$. Applying Theorem 2.2 we obtain

$$
\begin{gathered}
(\operatorname{det} B)(\operatorname{det} \hat{B})=\left[\left(\int_{\mathbb{R}^{2}} \mathrm{x}_{1}^{2}|\mathrm{f}|^{2} \mathrm{dx}\right)\left(\int_{\mathbb{R}^{2}} \mathrm{x}_{2}^{2}|\mathrm{f}|^{2} \mathrm{dx}\right)-\left(\int_{\mathbb{R}^{2}} \mathrm{x}_{1} \mathrm{x}_{2}|\mathrm{f}|^{2} \mathrm{dx}\right)^{2}\right] \\
\cdot\left[\left(\int_{\mathbb{R}^{2}} \mathrm{y}_{1}^{2}|\hat{\mathrm{f}}|^{2} \mathrm{dy}\right)-\left(\int_{\mathbb{R}^{2}} \mathrm{y}_{1} \mathrm{y}_{2}|\hat{\mathrm{f}}|^{2} \mathrm{dy}\right)^{2}\right] \geqslant\left(16 \pi^{2}\right)^{-2} .
\end{gathered}
$$

If we denote the bracketed terms above by $D\left[|f|^{2}\right]$ and $D\left[|\hat{f}|^{2}\right]$, the discrepancy of Schwarz's inequality, or the difference between variance and covariance of $|\mathrm{f}|^{2}$ and $|\hat{\mathrm{f}}|^{2}$, then the two dimensional Heisenberg-Weyl inequality shows that the discrepancies of $|\hat{\mathrm{f}}|^{2}$ and $|\hat{\mathrm{f}}|^{2}$ cannot both be small; $D\left[|\mathrm{f}|^{2}\right] D\left[|\hat{\mathrm{f}}|^{2}\right] \geqslant\left(16 \pi^{2}\right)^{-2}$.

A different generalization of (1.1) may be obtained along the lines of Theorem 1.1.

THEOREM 2.3. Let $\mathrm{f} \in \mathrm{S}\left(\mathbb{R}^{\mathrm{n}}\right)$, such that $\mathrm{f}\left(\mathrm{x}_{1}, \mathrm{x}_{2}, \ldots, \mathrm{x}_{n}\right)=0$, whenever $\mathrm{x}_{1}=0$ for some $i$. If $1<p \leqslant 2$ and $A(p)$ is the constant of (1.3), then

$$
\|\mathrm{f}\|_{2}^{2}<[2 \pi \mathrm{p} A(\mathrm{p})]^{\mathrm{n}}\left\|\mathrm{x}_{1} \ldots \mathrm{x}_{\mathrm{n}} \mathrm{f}\right\|\left\|_{\mathrm{p}}\right\| \mathrm{y}_{1} \ldots \mathrm{y}_{\mathrm{n}} \hat{\mathrm{f}} \|_{\mathrm{p}} \text {. }
$$

PROOF. We only give the proof for $n=2$ since the general case follows in exactly the same way. Let $f_{21}(x, y)=g(x, y)$, then

$$
f(x, y)=\int_{0}^{x} \int_{0}^{y} g(s, t) d t d s
$$

and by Hölder's and the two dimensional Hardy inequality, with $\mathbb{R}_{+}^{2}=(0, \infty) \times(0, \infty)$,

$$
\begin{aligned}
& \left.\int_{\mathbb{R}_{+}^{2}}|f(x, y)|^{2} d x d y<\left.\underset{\mathbb{R}_{+}^{2}}{\left(\int_{+}|x y f(x, y)| P_{d x d y}\right)^{1 / P}\left(\int_{\mathbb{R}_{+}^{2}} \mid f(x, y) / x y\right.}\right|^{P^{\prime} d x d y}\right)^{1 / p^{\prime}} \\
& \leqslant \mathrm{p}^{2}\left(\int_{\mathbb{R}_{+}^{2}}|x y f(x, y)| P_{d x d y}\right)^{1 / P}\left(\int_{\mathbb{R}_{+}^{2}}\left|f_{21}(x, y)\right|^{P^{\prime}} d x d y\right)^{1 / p^{\prime} .}
\end{aligned}
$$

On applying this estimate four times we obtain with $\mathrm{d} \mu=\mathrm{dxdy}$

$$
\| f||_{2}^{2}=\int_{\mathbb{R}_{+}^{2}}\left(|f(x, y)|^{2}+|f(x,-y)|^{2}+|f(-x,-y)|^{2}+|f(-x, y)|^{2}\right) d \mu
$$




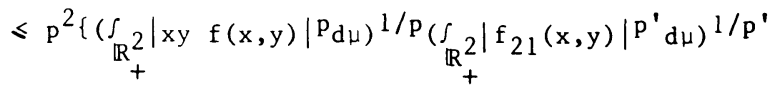

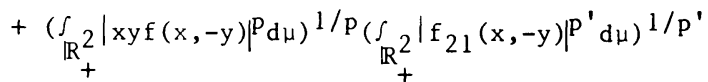

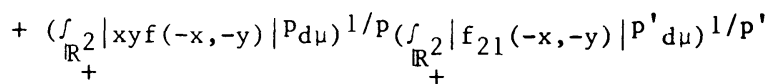

$$
\begin{aligned}
& +\left(\int_{\mathbb{R}_{+}^{2}}|\mathrm{xyf}(-\mathrm{x}, \mathrm{y})|^{\mathrm{P}} \mathrm{d} \mu\right)^{1 / \mathrm{P}}\left(\int_{\mathbb{R}_{+}^{2}}\left|\mathrm{f}_{21}(-\mathrm{x}, \mathrm{y})\right|^{\left.\left.\mathrm{P}^{\prime} \mathrm{d} \mu\right)^{1 / \mathrm{p}^{\prime}}\right\}}\right. \\
& \leqslant p^{2}\left\{\left(\int_{\mathbb{R}_{+}^{2}}|x y| P\left[|f(x, y)|^{P}+|f(x,-y)|^{P}+|f(-x,-y)|^{P}+\mid f(-x, y)\right)^{P}\right] d u\right)^{1 / P} \\
& x\left(\int_{\mathbb{R}_{+}^{2}}\left|f_{21}(x, y)\right| P^{\prime}+\left|f_{21}(x,-y)\right| p^{\prime}\right. \\
& \left.\left.\left.+\left|f_{21}(-x,-y)\right| P^{\prime}+\left|f_{21}(-x, y)\right|^{P^{\prime}}\right] d \mu\right)^{1 / p^{\prime}}\right\}
\end{aligned}
$$

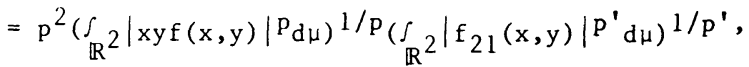

where the last inequality follows from Hölder's inequality. But by the sharp form of the Hausdorff-Young inequality with $n=2$ we obtain $\|f\|_{2}^{2}<[p A(p)]^{2} \| x y f||_{p}|| \hat{f}_{21}||_{p}$. Since $\left(\hat{i}_{-1}\right)(s, t)=4 \pi^{2} s t \hat{f}(s, t)$ the result follows.

3. WEIGHTED HIRSCHMAN ENTROPY INEQUALITY AND WEIGHTED HEISENBERG-WEYL INEQUALITY.

The results of the last section show that the Heisenberg-Weyl inequality is a consequence of the Hausdorff-Young theorem. Recently a number of weighted HausdorffYoung inequalities have been obtained [5], [6], [7] and [8]. We shall use these results in this section to obtain a weighted Hirschman entropy inequality as well as weighted form of the Heisenberg-Weyl inequality. Here we consider weighted extensions in $\mathbb{R}^{1}$ only.

Recall that if $g$ is a Lebesgue measurable function on $\mathbb{R}$, then the equi-measurable decreasing rearrangement of $g$ is defined by $g^{*}(t)=\inf \{y>0:|\{x \in \mathbb{R}:|g(x)|>y\}| \leqslant t\}$, where $y>0$ and $|E|$ denotes Lebesgue measure of the set $E$. Clearly, if $g$ is an even function on $\mathbb{R}$, decreasing on $(0, \infty)$, then for $t>0, g^{*}(t)=g(t / 2)$. We shall use this fact below.

DEFINITION 3.1. Let $\mathrm{u}$ and $\mathrm{v}$ be locally integrable functions of $\mathbb{R}$. We write $(\mathrm{u}, \mathrm{v}) \varepsilon \mathrm{F}_{\mathrm{p}, \mathrm{q}}^{*}, 1 \leqslant \mathrm{p} \leqslant \mathrm{q}<\infty$, if

$$
\sup \left(\int_{0}^{s}\left[u^{*}(t)\right]^{q} d t\right)^{1 / q}\left(\int_{0}^{1 / s}\left[(1 / v)^{*}(t)\right]^{p^{\prime}} d t\right)^{1 / p^{\prime}<\infty,}
$$

where in the case $p=1$ the second integral is replaced by the essential supremum of $(1 / \mathrm{v})^{*}(t)$ over $(0,1 / s)$.

If $\mathrm{u}$ and $1 / \mathrm{v}$ are even and decreasing on $(0, \infty)$ then $(3.1)$ is equivalent to

$$
\sup _{s>0}\left(\int_{0}^{s / 2}[u(x)]^{q} d x\right)^{1 / q}\left(\int_{0}^{1 /(2 s)} v(x)^{-p^{\prime}} d x\right)^{1 / p^{\prime}<\infty}
$$

and in this case we write $(u, v) \varepsilon F_{p, q}$.

The weighted Hausdorff-Young inequality is given in the following theorem:

THEOREM 3.1. ([ 5 ; Theorem 1.1]). Suppose $(u, v) \varepsilon F_{p, q}^{*}, 1 \leqslant p \leqslant q<\infty$ and $f \in L_{v}^{P}$. (i) If $\lim _{n \rightarrow \infty}|| f_{n}-\left.f\right|_{p, v}=0$ for a sequence of simple functions, then $\left\{\hat{f}_{n}\right\}$ converges in $L_{u}^{q}$ to a function $\hat{f} \varepsilon L_{u}^{q}$. $\hat{f}$ is independent of the sequence $\left\{\hat{f}_{n}\right\}$ and is called 
the Fourier transform of $f$.

(ii) there is a constant $B>0$ such that for all f $\varepsilon L_{V}^{p}$

$\|\hat{\mathrm{f}}\|_{\mathrm{q}, \mathrm{u}}<\mathrm{B}|| \mathrm{f}_{\mathrm{p}, \mathrm{v}}$.

(iii) If $\mathrm{g} \in \mathrm{L}_{1 / \mathrm{u}}^{\mathrm{q}}, \mathrm{q}>1$, then Parseval's formula

$$
\int_{\mathbb{R}} \hat{\mathrm{f}}(\mathrm{y}) \mathrm{g}(\mathrm{y}) \mathrm{dy}=\int_{\mathbb{R}} \mathrm{f}(\mathrm{t}) \hat{\mathrm{g}}(\mathrm{t}) \mathrm{dt}
$$

holds.

We note ([5], [6], [8]) that Theorem 3.1 is sharp in the sense that if $u$ and $v$ are even and satisfy (3.3), then ( $u, v)$ satisfies (3.2). The constant B in (3.3) is not sharp, however it is of the form $B=k . C$ where $k=k(p, q)$ is independent of $u$ and $v$ and $C$ is the supremum of (3.1), and in the case $u, 1 / v$ decreasing and even the supremum $(3.2)$.

A special case of Theorem 3.1 is the following:

COROLLARY 3.1. Suppose $\mathrm{f} \varepsilon \mathrm{L}_{\mathrm{v}}^{\mathrm{p}} 1 / 2 / \mathrm{p},\left(\mathrm{u}^{1-2 / \mathrm{p}^{\prime}}, \mathrm{v}^{1-2 / \mathrm{p}}\right) \varepsilon \mathrm{F}_{\mathrm{p}, \mathrm{p}^{\prime}}^{*}, 1<\mathrm{p}<2$, where $u$ and $v$ are even, decreasing as $(0, \infty)$ then

$$
\left(\int_{\mathbb{R}} \mathrm{u}(\mathrm{y})^{\mathrm{p}^{\prime}-2}|\hat{\mathrm{f}}(\mathrm{y})|^{\mathrm{P}^{\prime}}<\mathrm{k} \cdot \mathrm{C}_{\mathrm{p}}\left(\int_{\mathbb{R}} \mathrm{v}(\mathrm{x})^{\mathrm{p}-2}|\mathrm{f}(\mathrm{x})| \mathrm{P}_{\mathrm{dx}}\right)^{1 / \mathrm{p}}\right.
$$

where

$$
C_{p}=\sup _{s>0}\left(\int_{0}^{s / 2} u(x)^{p^{\prime}-2} d x\right)^{1 / p^{\prime}}\left(\int_{0}^{1 /(2 s)} v(x)(2-p) p^{\prime} / p_{d x}\right)^{1 / p^{\prime}} .
$$

Utilizing the last result we now give a weighted form of Hirschman's entropy inequality.

PROPOSITION 3.1. Suppose $f \in \mathrm{L}^{2} \cap \mathrm{L}_{\mathrm{l} / \mathrm{v}}$, where $\mathrm{u}$ and $\mathrm{v}$ satisfy the conditions of Corollary 3.1. If $\|\mathrm{f}\|_{2}=1$ and (3.4) holds with $0<\mathrm{k} \leqslant 2$ and $\mathrm{C}_{\mathrm{p}}$ remains bounded as $p \rightarrow 2$, then

$$
\begin{aligned}
& \int_{\mathbb{R}}|\hat{\mathrm{f}}(\mathrm{y})|^{2} \log |\mathrm{u}(\mathrm{y}) \hat{\mathrm{f}}(\mathrm{y})|^{2} \mathrm{dy}+\int_{\mathbb{R}}|\mathrm{f}(\mathrm{x})|^{2} \log |\mathrm{v}(\mathrm{x}) \mathrm{f}(\mathrm{x})|^{2} \mathrm{dx} \\
& \leqslant 2 \log \mathrm{k}+8 \sup _{\mathrm{s}>0}\left(\int_{0}^{\mathrm{s} / 2} \int_{0}^{1 /(2 s)} \log |\mathrm{u}(\mathrm{x}) \mathrm{v}(\mathrm{y})| \mathrm{dxdy}\right) .
\end{aligned}
$$

PROOF. Since $\mathrm{f} \in \mathrm{L}_{\mathrm{v}}^{\mathrm{p}} 1-2 / \mathrm{p}, 1<\mathrm{p}<2$, we apply Corollary 3.1 with $\mathrm{p}=2-\mathrm{r}, \mathrm{r}>0$, $p^{\prime}=2-r^{\prime}, r^{\prime}<0$ and $d \hat{\mu}(y)=|\hat{f}(y)|^{2} d y, d \mu(x)=|f(x)|^{2} d x$. Then (3.4) has the form

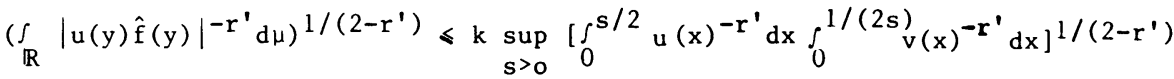

$$
\begin{aligned}
& \text { - }\left(\int_{\mathbb{R}}|\mathrm{v}(\mathrm{x}) \mathrm{f}(\mathrm{x})|^{-\mathrm{r}} \mathrm{d} \mu\right)^{1 /(2-r)}
\end{aligned}
$$

or, on raising the inequality ot the power $\left(2-r^{\prime}\right)\left(-1 / r^{\prime}\right)$, equivalently

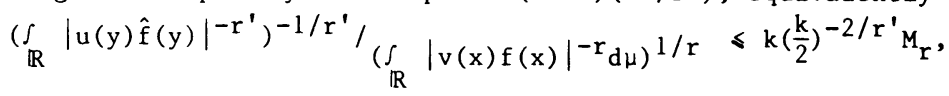

where

$$
M_{r}=\sup _{s>0}\left[f_{0}^{s / 2} \int_{0}^{1 /(2 s)}[u(x) v(y)]^{-r^{\prime}} 4 d x d y\right]^{-1 / r^{\prime}}
$$

Given $\varepsilon>0$ there is an $s_{0}>0$ such that

$$
M_{r} \leqslant\left[\int_{0}^{s_{0} / 2} f^{1 /(2 s)}[u(x) v(y)]^{-r^{\prime}} r d x d y\right]^{-1 / r^{\prime}}+\varepsilon
$$

so that 


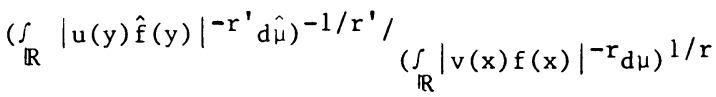

$$
\begin{aligned}
& \leqslant k\left(\left[\int_{0}^{s_{0} / 2} \int_{0}^{1 /\left(2 s_{0}\right)}[u(x) v(y)]^{-r^{\prime}} 4 d x d y\right]^{-1 / r^{\prime}}+\varepsilon\right),
\end{aligned}
$$

where we used the fact that $k / 2 \leqslant 1$. Now as $r \rightarrow 0+, r^{\prime} \rightarrow \sigma$, then on applying (2.1) to both sides of (3.5) we obtain

$$
\begin{aligned}
& \exp \left(\int_{\mathbb{R}} \log |u(y) \hat{f}(y)| d \hat{\mu}\right) / \exp \left(\int_{\mathbb{R}} \log |1 /[v(x) f(x)]| d \mu\right) \\
& \leqslant k\left[\exp \int_{0}^{s} s_{0} / 2 \int_{0}^{l /\left(2 s_{0}\right)} \log [v(y) u(x)] 4 d x d y+\varepsilon\right] \\
& \leqslant k\left[\exp \sup _{s>0} \int_{0}^{s / 2} 1 /(2 s) \log [u(x) v(y)] 4 d x d y+\varepsilon\right] .
\end{aligned}
$$

But $\varepsilon>0$ is arbitrary so that on taking logarithms we have

$$
\begin{gathered}
\frac{1}{2} \int_{-\infty}^{\infty}|\hat{\mathrm{f}}(\mathrm{y})|^{2} \log |\mathrm{u}(\mathrm{y}) \hat{\mathrm{f}}(\mathrm{y})|^{2} \mathrm{dy}+\frac{1}{2} \int_{-\infty}^{\infty}|\mathrm{f}(\mathrm{x})|^{2} \log |\mathrm{v}(\mathrm{x}) \mathrm{f}(\mathrm{x})|^{2} \mathrm{dx} \\
\leqslant \log \mathrm{k}+4 \sup _{\mathrm{s}>0}\left(\int_{0}^{s / 2} \log \mathrm{u}(\mathrm{x}) \mathrm{dx}+\int_{0}^{1 /(2 s)} \log v(\mathrm{y}) \mathrm{dy}\right)
\end{gathered}
$$

which yields the result.

Note that if $\mathrm{u}=\mathrm{v} \equiv \mathrm{l}$ and if $\mathrm{k} \leqslant \sqrt{2 / \mathrm{e}}$ we obtain (2.2) with $\mathrm{n}=1$.

We can write the conclusion of Proposition 3.1 in the form

$$
\begin{aligned}
E\left[|f|^{2}\right]+E\left[|\hat{f}|^{2}\right] & \leqslant 2 \log k+8 \sup _{s>0}\left(\int_{0}^{s / 2} \int_{0}^{l /(2 s)} \log |u(x) v(y)| d x d y\right) \\
& -\int_{\mathbb{R}}|\hat{f}(y)|^{2} \log |u(y)|^{2} d y-\int_{\mathbb{R}}|f(x)|^{2} \log |v(x)|^{2} d x .
\end{aligned}
$$

But since $([2]) E\left[|f|^{2}\right] \geqslant-\frac{1}{2}-\frac{1}{2} \log \left(2 \pi V\left[|f|^{2}\right]\right)$ and also with $f$ replaced by $\hat{f}$ we obtain another uncertainty inequality

$$
\begin{aligned}
V\left[|\mathrm{f}|^{2}\right] V\left[|\hat{\mathrm{f}}|^{2}\right] & \geqslant \frac{\mathrm{k}^{-4}}{4 \pi^{2} \mathrm{e}^{2}} \exp \left[-16 \sup _{\mathrm{s}>0} \int_{0}^{s / 2} \int_{0}^{1 /(2 s)} \log |\mathrm{uv}| \mathrm{dxdy}\right] \\
& x \exp \left(2 \int_{\mathbb{R}}|\hat{\mathrm{f}}|^{2} \log |\mathrm{u}|^{2} \mathrm{dy}\right) \exp \left(2 \int_{\mathbb{R}}|\mathrm{f}|^{2} \log |\mathrm{v}|^{2} \mathrm{dx}\right) .
\end{aligned}
$$

If $\mathrm{u}=\mathrm{v} \equiv \mathrm{l}$ and $\mathrm{k}=\sqrt{2 / \mathrm{e}}$ in this estimate we obtain (1.1).

THEOREM 3.2. (Heisenberg-Wey1 inequality). If $(1 / \mathrm{u}, \mathrm{v}) \varepsilon \mathrm{F}_{\mathrm{p}, \mathrm{q}}^{\star}, 1 \leqslant \mathrm{p} \leqslant \mathrm{q}<\infty$ and $f \in S(\mathbb{R})$, then

$$
\| \mathrm{f}||_{2}^{2}<\mathrm{C}\left(\int _ { \mathbb { R } } | \mathrm { u } ( \mathrm { x } ) \mathrm { xf } ( \mathrm { x } ) | ^ { q ^ { \prime } \mathrm { dx } ) } { } ^ { 1 / \mathrm { q } ^ { \prime } } \left(\int_{\mathbb{R}}|\mathrm{v}(\mathrm{y}) \mathrm{y} \hat{\mathrm{f}}(\mathrm{y})|^{\left.\mathrm{P}_{\mathrm{dy}}\right)^{1 / \mathrm{p}}}\right.\right.
$$

PROOF. Integration by parts and Hölder's inequality show that for $1 \leqslant q<\infty$ $\left\|\left.f\right|_{2} ^{2} \leqslant 2 \int_{\mathbb{R}}|x|\left|f(x) \| f^{\prime}(x)\right| d x\right.$

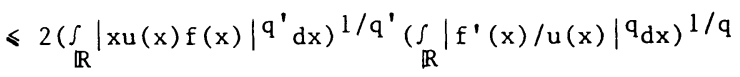

$$
\begin{aligned}
& \leqslant 2 C\left(\int_{\mathbb{R}}|\mathrm{xu}(\mathrm{x}) \mathrm{f}(\mathrm{x})|^{\left.\mathrm{q}^{\prime} \mathrm{dx}\right)}\right)^{1 / \mathrm{q}^{\prime}}\left(\int_{\mathbb{R}}\left|\mathrm{v}(\mathrm{y}) \hat{\mathrm{f}}^{\prime}(\mathrm{y})\right| \mathrm{P}_{\mathrm{dx}}\right)^{1 / \mathrm{p}},
\end{aligned}
$$

where the last inequality follows from (3.3). Since $\hat{f}^{\prime}(y)=2 \pi i y \hat{f}(y)$ the result follows.

Note that the case $\mathrm{p}=1$ also holds, provided the second integral in the $\mathrm{F}_{\mathrm{p}, \mathrm{q}}^{*}$ condition is interpreted as the essential supremum of $(1 / \mathrm{v})^{*}$ over $(0,1 / \mathrm{s})$. 
The same result holds also if we take $(1 / \mathrm{u}, \mathrm{v}) \varepsilon \mathrm{F}_{\mathrm{p}, \mathrm{q}}$

Observe also that the case $u=v \equiv 1$ and $q=p^{\prime}, 1<p \leqslant 2$ reduces to ( 1.4$)$, but with a different constant.

Weighted inequalities of the form (3.6) were also obtained by Cowling and Price

[3] but by quite different methods.

REFERENCES

1. HIRSCHMAN, I.I. A note on Entropy; Amer. J. Math. 79 (1957), 152-156.

2. SHANNON, C.E. and WEAVER, W. The Mathematical Theory of Communication; Univ. of Illinois, Urbana 1949.

3. BECKNER, W. Inequalities in Fourier Analysis; Annals of Math. (2), 102 (1975), (1), 159-182.

4. HARDY, G.H., LITTLEWOOD, J.E. and POLYA, G. Inequalities; Cambridge Univ. Press, 1959.

5. BENEDETTO, J.J., HEINIG, H.P. and JOHNSON, R. Boundary Values of Functions in Weighted Hardy Spaces; (preprint).

6. HEINIG, H.P. Weighted Norm Inequalities for Classes of Operators; Indiana U. Math. J. 33(4), (1984) 573-582.

7. JURKAT, W.B. and SAMPSON, G. On Rearrangement and Weight Inequalities for the Fourier Transform; Indiana U. Math. J. 32(2), 257-270.

8. MUCKENHOUPT, B. Weighted NOrm Inequalities for the Fourier Transform; Trans. A.M.S. 276 (1983), 729-742.

9. HEWITT, E. and STROMBERG, K. Real and Abstract Analysis; Springer Verl., NY 1965.

10. COWLING, M.G. and PRICE, J.F. Bandwidth Versus Time Concentration; The HeisenbergPauli-Weyl Inequality (Preprint). 


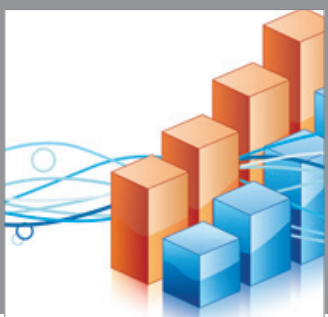

Advances in

Operations Research

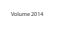

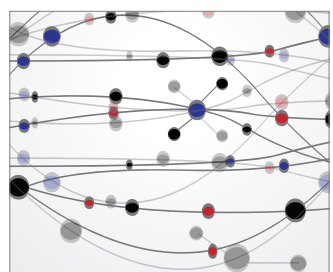

\section{The Scientific} World Journal
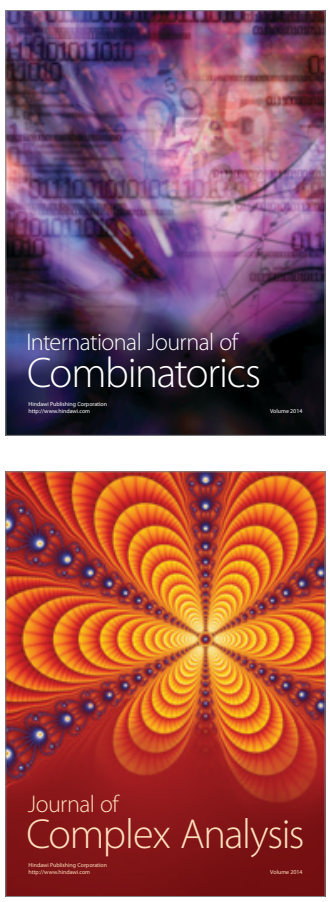

International Journal of

Mathematics and

Mathematical

Sciences
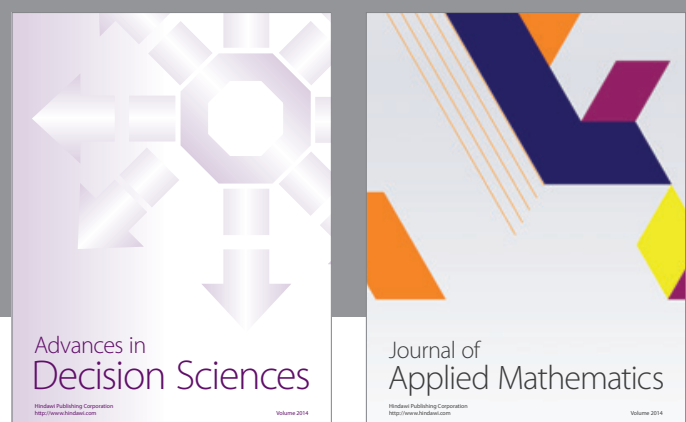

Journal of

Applied Mathematics
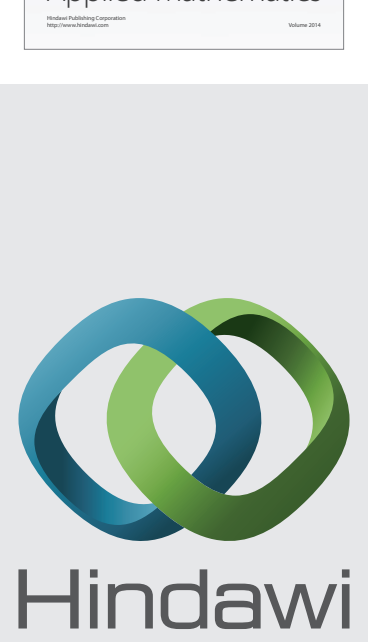

Submit your manuscripts at http://www.hindawi.com
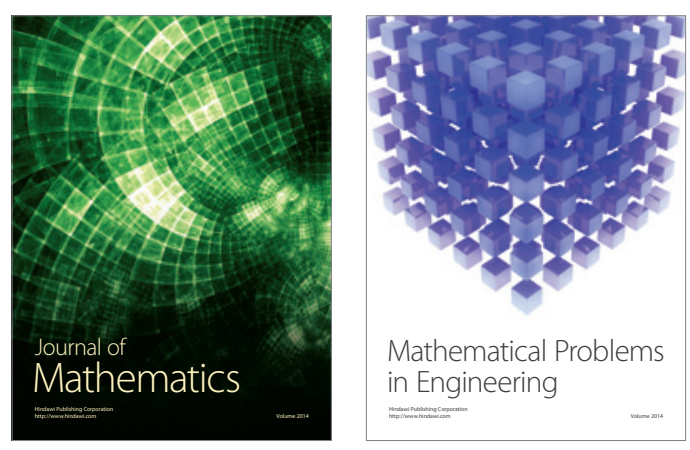

Mathematical Problems in Engineering
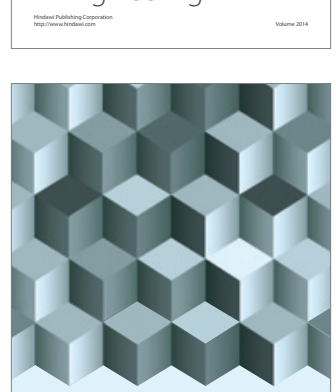

Journal of

Function Spaces
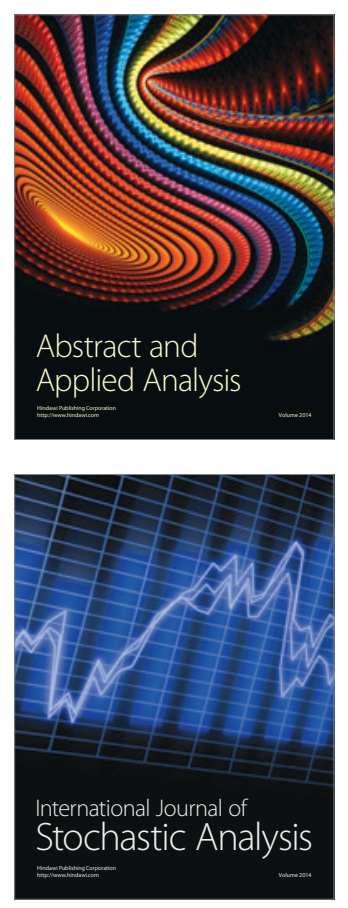

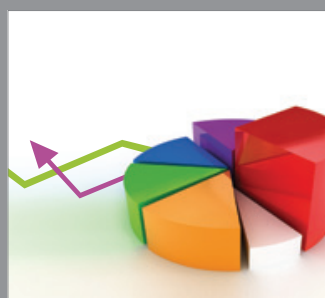

ournal of

Probability and Statistics

Promensencen
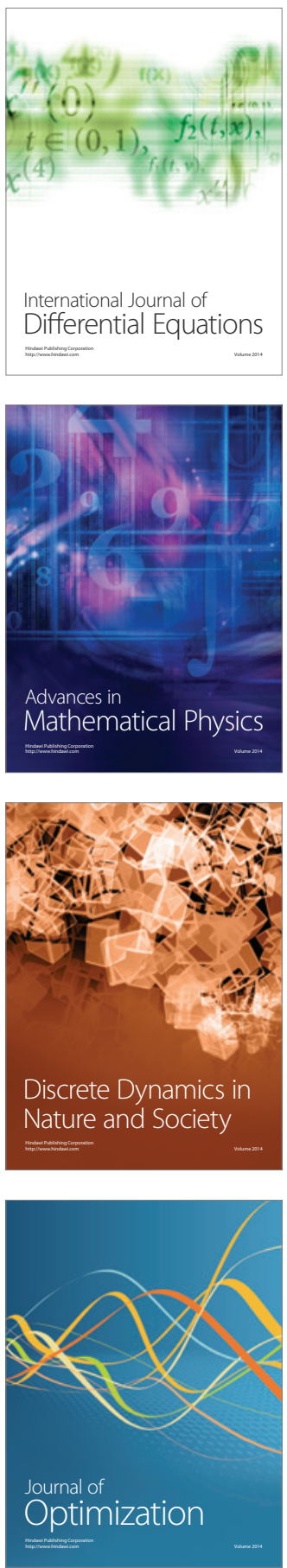